\title{
EDUCACIÓN EN SOSTENIBILIDAD Y PROMOCIÓN DE VOCACIONES CIENTÍFICO-TÉCNICAS EN ESTUDIANTES PREUNIVERSITARIOS A TRAVÉS DE LA CONSTRUCCIÓN DE UN VEHÍCULO SOLAR
}

\author{
Yolanda Ceada-Garrido, Antonio Javier Barragán, Juan Manuel Enrique, Ramón Tirado, José Manuel Andújar \\ yolanda.ceada@sc.uhu.es, \{antonio.barragan, juanm.enrique, andujar\}@diesia.uhu.es, rtirado@uhu.es
}

\section{Resumen}

Las politicas de sostenibilidad deben tener un fuerte componente educativo, ya que es importante que prevalezcan en el tiempo y sean aceptadas como parte de la realidad de las nuevas generaciones. El necesario cuidado del medio ambiente ha abierto una ventana de oportunidades para ingenieros y cientificos, ya que la gran mayoría de los productos deben ser rediseñados o adaptados a un nuevo marco regulatorio más estricto con su cuidado. Estas nuevas oportunidades requieren ingenieros cualificados, sin embargo, vemos como las titulaciones técnicas tienen cada vez menos alumnos. Para corregir esta contradicción, los autores han ideado un proyecto que consiste en la construcción de un vehículo solar por parte de estudiantes preuniversitarios, para posteriormente realizar un concurso público en el que se premiarán l.os mejores proyectos. De esta manera, los autores pretenden motivar a estos estudiantes para que se matriculen en carreras técnicas, a la vez que promueven la conciencia ecológica y dan visibilidad a la labor social y educativa de los grupos de investigación universitarios. En este trabajo se describe el proyecto realizado en la Universidad de Huelva junto con 15 institutos en 2018. Respecto al artículo publicado en las Jornadas de Automática de 2018, en esta versión se incluyen los resultados obtenidos en el mismo a partir de encuestas realizadas a los participantes.

Palabras clave: Educación, Energía solar, Estudiantes, Motivación, Movilidad sostenible, Vehículo eléctrico.

\section{INTRODUCCIÓN}

Los problemas de sostenibilidad son altamente complejos, sistémicos y no pueden ser fácilmente remediados[1]. Las emisiones de los vehículos constituyen una de las principales fuentes de contaminación gaseosa y de partículas del aire en las zonas urbanas, y son una fuente esencial de contaminación atmosférica en las zonas metropolitanas[2]. Representa alrededor del 15-50\% de la masa total de partículas finas en las ciudades[3]. La educa- ción es un factor determinante para el progreso y el desarrollo de las sociedades[4]. Por lo tanto, las acciones educativas, así como la investigación, son tareas necesarias para avanzar hacia una sociedad sostenible. Sin embargo, estamos viendo como las titulaciones técnicas tienen cada vez menos alumnos.

El objetivo del proyecto Carrera Urbana Sostenible (SUR) es aumentar el interés por la ciencia y la tecnología entre los estudiantes preuniversitarios. SUR ha sido concebido dentro del grupo de investigación Çontrol y Robótica" de la Universidad de Huelva (TEP-192), y ha sido financiado por la Fundación Española para la Ciencia y la Tecnología (FECYT), del Ministerio de Ciencia, Innovación y Universidades.

La idea principal de SUR es animar a los estudiantes preuniversitarios de los institutos del suroeste de la península a estudiar titulaciones científicotécnicas. Asimismo, también se pretende llevar la ciencia, la tecnología y la innovación a estudiantes no universitarios y a la sociedad en general. En este proyecto, los estudiantes deben crear un vehículo eléctrico que utilice energía solar. Este vehículo deberá ser capaz de transportar al menos a una persona en un entorno urbano. La organización proporciona a los centros participantes un kit de desarrollo y propone a los participantes la aplicación del Aprendizaje Basado en Proyectos (PBL por sus siglas en inglés) para incluir el proyecto SUR en sus clases diarias. PBL ha demostrado su eficacia para el aprendizaje, especialmente en materias científico-técnicas $[5,6]$. El modelo $\mathrm{PBL}$, que se basa en el estudio de proyectos, es un enfoque que sitúa a los alumnos en el centro del proceso de aprendizaje y los prepara para la vida actual exponiéndolos a problemas reales que deben resolver [4]. PBL se ha convertido en un enfoque educativo de referencia, sobre el que existe cada vez más documentación y estudios debido a la importancia que ha tomado en los últimos años de la educación centrada en el estudiante [7]. Además de las propias competencias ingenieriles, se desarrollan competencias transversales como la creatividad, la capacidad de colaboración, la toma de decisiones, la organización del trabajo y la 
gestión del tiempo, la capacidad de investigación y desarrollo de soluciones, la conciencia ecológica y el espíritu emprendedor, así como la autoestima del alumno [5]. El proyecto SUR también acerca la ciencia y la tecnología a grupos sociales que normalmente están muy alejados de las acciones de divulgación científica, lo que le añade un componente social innovador.

Este trabajo se divide en las siguientes secciones: después de la introducción se explica en detalle la metodología utilizada en el proyecto. A continuación se presentan los resultados de la experiencia. Finalmente, se discuten algunas conclusiones y posibles mejoras.

\section{Método}

El aprendizaje basado en proyectos es un elemento clave para adquirir conocimiento a través de la experimentación [8]. En este proyecto los estudiantes deben construir un vehículo eléctrico que utilice energía solar, el cual debe ser capaz de transportar al menos a una persona en un entorno urbano. El proyecto implica casi un curso de trabajo, tanto para profesores como para estudiantes, por lo que es importante una buena organización del mismo. Con el fin de asegurar el mayor éxito posible entre los participantes y obtener toda la información relevante al respecto, el proyecto se divide en tres fases: conferencias técnicas, construcción/competición de vehículos y evaluación. A continuación se detalla cada una de estas fases.

\subsection{Conferencia técnica}

Las sesiones técnicas, celebradas en la Universidad de Huelva, fueron el punto de partida del proyecto y un primer contacto con todos los participantes. Aproximadamente asistieron 50 representantes de varios centros de la provincia de Huelva, un centro de Badajoz y 2 centros de Faro (Portugal). Durante este acto se presentó el reglamento de la edición 2018, se estableció un debate con los asistentes sobre las pruebas a realizar, los métodos de puntuación y los posibles detalles a mejorar respecto a la edición anterior (2017). Este debate fue muy enriquecedor, y se tomaron algunas ideas para incorporarlos a la nueva normativa de 2018 . Muchos de los centros presentes se inscribieron en el concurso en ese momento.

\subsection{Construcción del vehículo y competición}

La fase de construcción del vehículo es la más duradera del proyecto. Durante esta fase los participantes deben crear un vehículo de 3 ó 4 ruedas, e integrar en él un motor eléctrico, dos paneles solares y la electrónica asociada. Además, deben resolver los retos técnicos propuestos por la organización, como por ejemplo la realización de un sistema de telemetría (este fue uno de los retos de la edición 2018).

Esta fase comienza con la visita de los técnicos del proyecto a cada centro participante para asesorar a los profesores en el montaje del motor, los paneles solares y su electrónica asociada y un medidor de energía. Este material es entregado a los centros por la organización. El medidor permite evaluar la eficiencia energética del vehículo, que será necesaria para una de las pruebas de la competición. Estas visitas también se utilizan para resolver dudas de forma más personalizada. El personal técnico pertenece al Grupo de Investigación en Control y Robótica (TEP-192) de la Universidad de Huelva y es especialista en estos campos.

Cada centro llevó a cabo la construcción del vehículo de manera desigual. Cada profesor optó por la organización del proyecto en función de sus consideraciones personales, el interés de sus alumnos y el centro. Algunos comenzaron inmediatamente e incluyeron la construcción del vehículo como parte de las propias asignaturas (básicamente los centros de formación profesional); mientras que otros profesores prefirieron abordar el proyecto como una actividad extracurricular para los estudiantes interesados (voluntarios). Esta disparidad fue conocida y anticipada por la organización a través de la experiencia de ediciones anteriores, y creemos que es comprensible dada la diferencia de nivel e integración del proyecto en los diferentes centros participantes.

Con el objetivo de mejorar el aprendizaje de los estudiantes sobre las tecnologías, y aprovechar el proyecto para aumentar sus posibles vocaciones hacia una educación técnica, la organización propone a los participantes la aplicación de PBL para introducir el proyecto SUR en sus clases diarias. PBL es un método sistemático de enseñanza y aprendizaje, que involucra a los estudiantes en tareas complejas y reales que resultan en un producto o presentación a una audiencia, permitiéndoles adquirir conocimientos y habilidades para mejorar su vida $[9]$.

Hasta la fecha del concurso, se realizaron varias visitas a los centros para conocer la evolución de sus proyectos. Durante estas visitas también se dio asesoramiento tanto técnico como pedagógico, y se resolvieron numerosos problemas con la construcción del vehículo, su conexión, la construcción de los cargadores de baterías, así como la programación de la plataforma de control utilizada (Arduino). 
El proyecto SUR concluyó con un concurso público de vehículos construidos por estudiantes, con alto impacto social. El 1 de junio de 2018 se celebró SUR18 en el Campus de El Carmen de la Universidad de Huelva. Este día todos los centros asistieron con sus prototipos, y otros como visitantes. Compitieron 13 equipos, ya que algunos equipos no terminaron sus prototipos a tiempo.

El jurado calificó la calidad del vehículo según: un proyecto y un vídeo entregado previamente (20\%), el diseño, la originalidad e innovación (30\%), la funcionalidad y robustez $(10 \%)$, la comerciabilidad $(10 \%)$, y el cumplimiento de 2 retos tecnológicos voluntarios propuestos por la organización ( $15 \%$ cada uno). Una vez que todos los centros pasaron la evaluación del jurado, se iniciaron las pruebas en pista. Se realizaron las siguientes pruebas: una prueba de eficiencia energética, una prueba de velocidad y una prueba de maniobrabilidad. El concurso finalizó con la entrega de premios y reconocimientos, y tuvo un fuerte impacto mediático en radio, prensa y televisión.

\subsection{Evaluación}

Con el fin de estudiar el impacto del proyecto en los estudiantes, se realizaron dos encuestas: una para los estudiantes y otra para los profesores. Un total de 123 estudiantes y 19 profesores de 10 centros distintos respondieron a las encuestas.

Sobre los profesores, se recogieron datos identificativos (como edad, sexo, años de experiencia, etc.), así como datos relacionados con la integración del proyecto en la programación del aula y el nivel de aprendizaje alcanzado por los alumnos (medido en competencias técnicas y transversales). Los datos recogidos sobre los estudiantes fueron: datos de identificación, competencias técnicas y transversales, andamiaje, enfoque/recursos y autodeterminación (ver Tabla 1 ).

Los resultados de las encuestas nos han ayudado a comprobar que el proyecto goza de una gran aceptación, tanto por parte de los profesores como de sus alumnos, y que es un proyecto motivador, como se puede comprobar en el siguiente apartado.

\section{Resultados}

La mayoría de los profesores participantes eran varones $(17 / 2)$, de entre 45 y 52 años de edad, con una experiencia docente de más de 10 años (aunque obviamente hay una amplia gama de edades y años de experiencia). En la mayoría de los centros el proyecto ha sido llevado a cabo por 2 profesores, con un solo centro en el que ha participado un solo profesor y otro en el que han participado 4 .
El proyecto ha abarcado prácticamente todos los niveles educativos preuniversitarios: Enseñanza Secundaria, Bachillerato y Ciclos Formativos de grado Medio y Superior. La mayoría de los profesores han optado por incluir el diseño del vehículo como parte de sus asignaturas, pero no de forma obligatoria, con una importancia desigual en el nivel de cualificación que aportaba el mismo a la nota final. La media de alumnos que han participando en los proyectos ha sido de 10 , y el nivel de abandono de los alumnos ha sido prácticamente insignificante.

Atendiendo a las competencias técnicas, la mayoría de los estudiantes opinan que han mejorado considerablemente sus habilidades en el manejo de herramientas, el diseño y construcción de circuitos electrónicos simples, y el diseño y construcción de máquinas fiables y robustas. Han mejorado considerablemente sus habilidades para instalar terminales de baterías, instalar paneles fotovoltaicos y conectarlos al controlador de carga, así como instalar cableado. Sorprendentemente para los organizadores, la mayoría cree que no han mejorado sus habilidades para aplicar los principios básicos de programación a través de la plataforma Arduino y para manejar software específico para el diseño de estructuras (CAD). Alcanzar estas habilidades era uno de los objetivo de los organizadores, por lo que este resultado requerirá un estudio y modificación del proyecto para futuras ediciones.

En cuanto a las competencias transversales, la mayoría de los estudiantes consideran que han mejorado mucho su iniciativa, su capacidad de emprender proyectos originales, su conciencia de la conservación del medio ambiente, y su capacidad de trabajar en equipo. También consideran que ha mejorado mucho su capacidad de organizar tareas a lo largo del tiempo, su capacidad de autoestudio y su capacidad persistir hasta lograr sus objetivos. Finalmente, las encuestas realizadas reflejan que también consideran que han mejorado significativamente sus habilidades matemáticas y sus habilidades básicas en ciencia y tecnología, así como su capacidad para la redacción de proyectos.

$\mathrm{Al}$ consultar a los alumnos sobre la forma de abordar el proyecto, se observa que se sienten muy implicados en la generación de nuevas ideas, en la designación del responsable, en el control del ritmo de trabajo, en la investigación y en la búsqueda de nuevas soluciones. Percibieron que tareas como describir el alcance del proyecto (materiales, requisitos y características) al principio del proyecto, descomponer el proyecto en fases durante la planificación, especificar los "módulos de trabajo" y especificar las actividades en cada "módulo" eran llevadas a cabo por ellos contando con la ayu- 

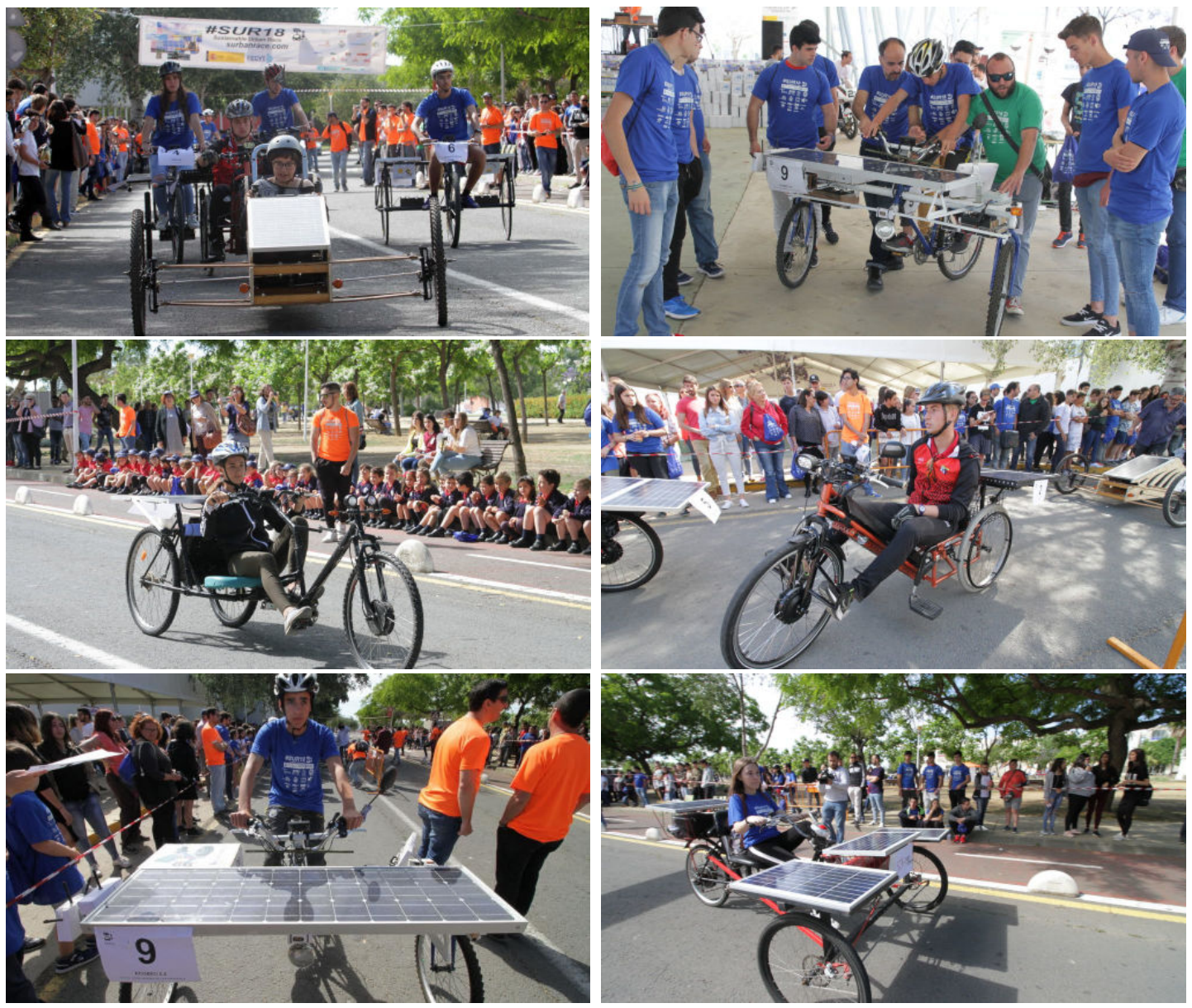

Figura 1: Algunas imágenes de la competición SUR de 2018.

da del profesor. Sin embargo, establecer la fecha de finalización de cada "módulo" o actividad y controlar el número y porcentaje de tareas realizadas, en curso o no iniciadas, fueron los aspectos en los que menos intervinieron; ya que las decisiones fueron tomadas directamente por el profesor en muchos de los casos.

La mayoría de los estudiantes percibieron el proyecto como un reto realista en el que el material fue presentado en detalle. Y muchos de ellos creen que los problemas que se plantearon son conceptos o principios centrales de la asignatura, que el proyecto es una parte central del currículo y que los componentes eran adecuados.

La mayoría de los estudiantes consideran muy satisfactoria la experiencia global del proyecto, la calidad del contacto con el profesor y la calidad del contacto con los miembros del equipo. También consideraron satisfactorias las directrices o consejos proporcionados por el instructor y las herramientas y plantillas para organizar el trabajo. A su vez, la mayoría percibió que el profesor les aconsejaba siempre que lo necesitaban, que eran ellos los que decidían cómo llevar a cabo las ac- tividades de grupo y que a menudo eran ellos los que tomaban las decisiones sobre cómo desarrollar el proyecto. Sin embargo, hay una disparidad de opiniones sobre si podrían dirigir el proyecto por sí mismos y si llevaron a cabo discusiones muy estimulantes.

En general, los resultados obtenidos por las encuestas vienen a certificar empíricamente la buena impresión que los organizadores tienen sobre los resultados del proyecto y el grado de implicación tanto de estudiantes como de docentes en el desarrollo del mismo.

\section{Discusión y conclusiones}

El resultado global del proyecto ha sido muy satisfactorio. El formato elegido es muy innovador e interesante, y representa un reto para los participantes, tanto estudiantes como profesores. A pesar del desafío, casi todos los centros logran completar con éxito su prototipo de vehículo. Hemos observado, y se ha comprobado a través de las encuestas realizadas, que el hecho de poder superar este reto aumenta enormemente la autoestima de los alum- 
Cuadro 1: Conceptos medidos en la encuestas.

\begin{tabular}{|l|l|}
\hline Profesores & Estudiantes \\
\hline $\begin{array}{l}\text { Datos demográficos: sexo, edad, experiencia do- } \\
\text { cente y número de profesores }\end{array}$ & $\begin{array}{l}\text { Datos de identificación: número de participan- } \\
\text { tes, etapa educativa, edad y sexo }\end{array}$ \\
\hline $\begin{array}{l}\text { Aspectos curriculares: nivel educativo, integra- } \\
\text { ción en el plan de estudios y número de estu- } \\
\text { diantes }\end{array}$ & $\begin{array}{l}\text { Resultados de aprendizaje: competencias técni- } \\
\text { cas y transversales adquiridas }\end{array}$ \\
\hline Competencias: técnicas y transversales & $\begin{array}{l}\text { Andamios durante el proyecto. Enfoque y re- } \\
\text { cursos }\end{array}$ \\
\hline & $\begin{array}{l}\text { Autodeterminación: autonomía para el traba- } \\
\text { jo en equipo, relaciones con los compañeros y } \\
\text { autoeficacia }\end{array}$ \\
\hline
\end{tabular}

nos. Esto también ayuda a ver la tecnología como algo interesante y útil.

Una consecuencia muy interesante del proyecto ha sido que los estudiantes han asociado la tecnología con la protección del medio ambiente. Normalmente se tiene la idea equivocada de que la tecnología es dañina para el medio ambiente, sin embargo, a través de este proyecto muchos estudiantes han entendido que los avances tecnológicos son los que permitirán una sociedad más sostenible.

Otro objetivo importante del proyecto es fomentar el deseo de estudiar ingeniería entre los estudiantes preuniversitarios. En base a las encuestas realizadas, el proyecto ha sido muy interesante para la mayoría de los estudiantes, por lo que esperamos que haya creado alguna vocación ingenieril entre ellos.

Otro de los objetivos de la edición de 2018 fue el aumento de la participación femenina en comparación con ediciones anteriores, donde era casi inexistente. Con una participación femenina de algo más de $1 / 3$, consideramos que las iniciativas tomadas han sido fructíferas, y continuaremos con la intención de aumentar este porcentaje en futuras ediciones.

Por supuesto, también se han detectado algunas cosas para mejorar. Por ejemplo, la necesidad de fomentar más la importancia de la programación en el proyecto, ya que los estudiantes han dado poca importancia a pesar de ser algo que los organizadores querían fomentar. Estos resultados nos ayudarán a planificar los retos y objetivos de la próxima edición con más información.

En conclusión, creemos que el proyecto ha cumplido los objetivos marcados de forma muy satisfactoria, ha tenido una gran aceptación entre los centros preuniversitarios de la región y ha tenido una gran repercusión mediática, tanto en prensa como en radio y televisión. Los organizadores esperan seguir mejorando en futuras ediciones y aumentar el número de participantes.

\section{Agradecimientos}

Este trabajo ha sido una contribución del proyecto Competición de vehículos solares para el fomento de las vocaciones científico- técnicas mediante el aprendizaje basado en proyectos - SUR19-20 (FCT-18-13386) financiado la Fundación Española para la Ciencia y la Tecnología (FECYT) del Ministerio de Ciencia, Innovación y Universidades.

\section{English summary}

EDUCATION IN SUSTAINABI-
LITY AND PROMOTION OF
SCIENTIFIC-TECHNICAL
CATIONS IN PRE-UNIVERSITY
STUDENTS THROUGH THE
CONSTRUCTION OF A SOLAR
VEHICLE

\section{Abstract}

Sustainability policies must have a strong educational component, since they must remain in time and be accepted as part of the reality of the new generations. The necessary care of the environment has opened a window of opportunities for engineers and scientists, since the vast majority of products must be redesigned or adapted to a new regulatory framework stricter with the care of the environment. These new opportunities require qualified engineers, however, we are seeing how technical qualifications have fewer and fewer students 
(at least in Spain). To correct this contradiction, the authors have devised a project that consists of the construction of a solar vehicle by pre-university students, to later carry out a public competition in which to reward the best projects. In this way, the authors intend to motivate these students to enroll in technical degrees, while promoting ecological awareness and giving visibility to the social and educational work of university research groups. This paper describes the project carried out at the University of Huelva together with 15 institutes in 2018, as well as some of the results obtained in it.

Keywords: Education, Electrical vehicle, Solar energy, Student, Sustainable mobility.

[1] Wiek, A., Ness, B., Schweizer-Ries, P., Brand, F. and Farioli, F.: From complex systems analysis to transformational change: a comparative appraisal of sustainability science projects. Sustain Sci 7, 5-24 (2012).

[2] Chunyi, L., Yilan, H., Huanhuan, G., Gaojie, W., Yifei, W., Wei, L. and Lijuan, C.: The Concentrations and Removal Effects of PM10 and PM2.5 on a Wetland in Beijing. Sustainability 11(5), 1312 (2019).

[3] Sheesley, R. J., Schauer, J. J., Zheng, M., and Wang, B.: Sensitivity of Molecular Marker-Based CMB Models to Biomass Burning Source Profiles. Atmospheric Environment 41 (39), 9050-9063 (2007).

[4] Tasc1, B. G.: Project Based Learning from Elementary School to College, Tool: Architecture. Procedia - Social and Behavioral Sciences 186, $770-775$ (2015).

[5] Mioduser, D., Betzer, N. (2007). The contribution of project-based learning to high achievers' acquisition of technological knowledge. International Journal of Techonology and Design Education, 18, 59-77.

[6] González, María Paz Sánchez. Técnicas docentes y sistemas de evaluación en Educación Superior. Vol. 26. Narcea Ediciones, 2010.

[7] Tasci, B. G.: Project Based Learning from Elementary School to College, Tool: Architecture. Procedia - Social and Behavioral Sciences 186, 770-775 (2015).
[8] Efstratia, D.: Experiential education through project based learning. Procedia - Social and Behavioral Sciences 152, 1256 - 1260 (2014).

[9] Chen, C - H. and Yang, Y - C.: Revisiting the effects of project-based learning on students' academic achievement: A meta-analysis investigating moderators. Educational Research Review 26, 71-81 (2019).

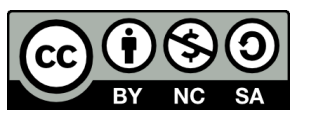
(C) 2019 by the authors. Submitted for possible open access publication under the terms and conditions of the Creative Commons Attribution CC BY-NC-SA 4.0 license (https://creativecommons.org/licenses/by-ncsa/4.0/deed.es). 